\title{
Regulation of fertilization in male rats by CatSper2 knockdown
}

\begin{abstract}
Zhen Zhang ${ }^{1}$, Gen-Lin Wang ${ }^{1}$, Hui-Xia Li ${ }^{1}$, Lian Li $^{1}$, Qun-Wei Cui ${ }^{1}$, Cheng-Bin Wei ${ }^{2}$ and Fei Zhou ${ }^{3}$
Interest in ion channels as drug targets for contraception has grown with the realization that certain ion channel subunits are located exclusively in sperm. Selective knockdown of ion channel subunits can lead to infertility without ill effects, and selective inhibitors and/ or openers of these ion channels could interfere with sperm function. In this study, in vivo electroporation (EP) and rete testis microinjection-mediated plasmid DNA were adopted to silence CatSper2 expression, which is essential in sperm hyperactivation. The results showed that high transfection efficiency and expression were achieved by plasmid DNA that was directly injected into the rete testis. As a result of the expression of CatSper2 being blocked, the treatment group showed significantly lower $(P<0.05)$ hyperactivation rate, fertilization rate in vitro, migration motility in viscoelastic solution and intracellular $\mathrm{Ca}^{2+}$ peak. The low hyperactivation and fertilization rates lasted for 60 days. Meanwhile, analysis of the sperm survival rate and testis histology indicated that in vivo EP had no significant effect on the function of the testis, spermatogenesis or sperm activity. The present study demonstrated that it was feasible to achieve male contraception by silencing the expression of CatSper2, the key protein involved in sperm hyperactivation.
\end{abstract}

Asian Journal of Andrology (2012) 14, 301-309; doi:10.1038/aja.2011.118; published online 17 October 2011

Keywords: Catsper2; in vivo electroporation; male contraception; rete testis microinjection

\section{INTRODUCTION}

Great advances in contraceptive research have been achieved in the past few decades, but there are still only a limited number of contraceptive measures or drugs available for men. ${ }^{1,2}$ Such limitations may be due to various factors including the difficulty in suppressing the generation of large numbers of sperm. Hormonal contraception that blocks sperm production by disrupting the hypothalamic-pituitarytesticular axis is currently recommended, but this approach still has some obstacles to overcome. ${ }^{2,3}$ One major hurdle is that complete hormonal suppression of spermatogenesis is necessary. Considering the drawbacks of hormonal methods, there is a growing interest in the development of non-hormonal methods for male contraception. Presently, several approaches are being pursued, ${ }^{4}$ including the development of gene vaccines for sperm surface antigens ${ }^{5}$ and drugs designed to cause retention of sperm in the epididymus. ${ }^{6}$ Chinese medicines such as gossypol and Tripterygium and other active ingredients have also received increased international research interest. ${ }^{4}$ The non-hormonal methods are appealing because of their minimal side effects, reversibility and because these methods work by means of agent or molecular regulation. ${ }^{4}$ This study focused on a non-hormonal method of molecular regulation.

Ion channels regulate the permeability of the plasma membrane and maintain the proper intracellular ionic environment, which is closely related to regulation of cell excitability, potential changes, cell secretion, cell volume maintenance and epithelial membrane electrolyte fluidity. ${ }^{7-10}$ With studies on ion channels progressing, researchers have generally agreed that ion channel regulation will be an important breakthrough of the next generation of contraceptives. ${ }^{11,12}$ Clarification of the role and function of the CatSper ion channel protein family suggests that it may be a viable drug target, which supports this prediction. ${ }^{11,13}$ As a unique ion channel that mediates $\mathrm{Ca}^{2+}$, the CatSper proteins play an important role in sperm hyperactivation and fertilization capacity. ${ }^{14}$ The CatSper family consists of four homologous proteins ${ }^{14}$ whose expressions are exclusively located in the tissue of testis and sperm at different levels. CatSper1 and CatSper2 proteins form a homodimer that is mainly located at the principal piece of the sperm tail flagellar plasma membrane. Lack of either protein will accelerate the frequency of sperm flagellar beat and diminish the amplitude of the beat, which ultimately causes failure of fertilization. ${ }^{13,15}$ The failure to achieve functional expression of CatSper 1 and CatSper2 singly or in conjunction with heterogonous expression systems suggests that CatSper1 and CatSper2 proteins require additional subunits and/or interaction partners to function. ${ }^{11,16}$ Previous studies have confirmed high expression of CatSper3 and CatSper4 in the sperm acrosome. Therefore, these proteins may play roles in regulating intracellular $\mathrm{Ca}^{2+}$ levels in the process of acrosome reaction. ${ }^{17}$ However, recent studies have demonstrated a possibility of a formed tetramer by the four family members to mediate sperm hyperactivation. ${ }^{18}$

In contrast to higher homology between CatSper3 (mouse vs. rat $61.1 \%$ ) and CatSper4 (mouse vs. rat 87.4\%), the homology between Catsper1 and Catsper2 is low; however, the function and structure of 
CatSper1 and CatSper2 are very similar. These two proteins are expressed at different levels along the flagellum. ${ }^{11,16}$ It has been shown that the levels of Catsper1 mRNA in ejaculated human spermatozoa could be a more feasible method for infertility screening than testis biopsy. Furthermore, it has been suggested that human CatSper1 could be a possible target for immunocontraception. ${ }^{19}$

In 1982, Neumann et al. ${ }^{20}$ imposed short but powerful electricity pulses on cells to transiently enhance transduction and permeability of the cells. This electroporation-mediated transfection of nucleotides or drugs into cells has developed into a very effective biological technique. $^{21,22}$ In recent years, a number of studies in mice have shown that it is possible to use this approach to express reporter genes such as lac $Z$ and green fluorescent protein (GFP) and its variants, under the control of constitutive or testis-specific gene promoters, in both spermatogenic cells ${ }^{23-28}$ and testicular sperm. ${ }^{25}$ These findings have led to the proposal that in vivo gene transfer into the testis provides an alternative way to study gene expression in testis and sperm, and to potentially explore male contraceptives. ${ }^{29}$

The purpose of this study was to verify the feasibility of using CatSper2-the critical protein in the sperm hyperactivation signalling pathway-as a target for male contraception. This was done by designing a target sequence according to the 785 sequence of the open reading frame of the CatSper2 subunit and inserting the target sequence into the U6 promoter expression vector. Electroporation-mediated transfection was performed in testis following rete testis microinjection in vivo. CatSper2 protein synthesis was blocked by continuous transcription of double-stranded short hairpin RNA (shRNA) in testis seminiferous tubules, which induced sterility in male rats.

\section{MATERIALS AND METHODS}

\section{Experiment animals}

Specific pathogen-free-class young male (Sprague-Dawley rats (licence No. SCXK-20080033) weighing between 300 and $400 \mathrm{~g}$ were purchased from the Experimental Animal Centre of Zhejiang Province, China, and were acclimatized in the Experimental Animal Centre of Nanjing Agricultural University, China. All the experimental procedures were in accordance with the rules of the Animal Experimental Committee of Nanjing Agricultural University. The experimental Sprague-Dawley rats were randomly divided into 3, 5, $9,13,22$ and 60 days treatment groups, as well as the negative control (NC) GFP group and a control group.

\section{Construction and synthesis of small interfering RNA (siRNA) plasmid vector}

Three targeting sequences for Catsper2 in the open reading frame were designed in vitro, as described in our previous paper. ${ }^{30}$ The results showed that Catsper2-785 was an optimal target sequence with the highest knockdown efficacy; therefore, Catsper2-785 was chosen as the only targeted sequence site in the in vivo electroporation (EP) and for plasmid construction (GeneBank accession No. NM_001012220.1). We designed Catsper2 shRNA oligonucleotides (sense, 5'-CACCGAATATTCCCGTTCAACTATTCAAGAGATAGTTGAACGGGAATATTCTTTTTTG-3'; antisense, 5' -GATCCAAAAAAGAATATTCCCGTTCAACTATCTCTTGAATAGTTGAACGGGAATATTC-3') to test. The NC shRNA oligonucleotides were designed to have no significant homology with CatSper2 (sense, 5'-CACCGTTCTCCGAACGTGTCACGTCAAGAGATTACGTGACACGTTCGGAGAATTTTTTG-3'; antisense, 5' -GATCCAAAAAATTCTCCGAACGTGTCACGTAATCTCTTGACGTGACACGTTCGGAGAAC-3'). These oligonucleotides were annealed to generate double-stranded DNA and were ligated into the linearized empty vector pGPU6/GFP/Neo. The siRNA plasmids encoded shRNAs that specifically targeted CatSper2 mRNA. The shRNAs had no significant homology with other known genes. The nucleotide sequences of CatSper2 siRNAs (pGPU6/GFP/ Neo-CatSper2) were verified by automated DNA sequencing. All of the plasmids were purchased from Genepharma (Shanghai, China), multiplied in the DH5 $\alpha$ strain of Escherichia coli and purified using Qiagen Mega Kits (Qiagen, Shanghai, China). All other reagents were of analytical grade.

\section{In vivo electroporation and detection of transgene expression}

Male Sprague-Dawley rats were anaesthetized by intraperitoneal injection of pentobarbital sodium $\left(75 \mathrm{mg} \mathrm{kg}^{-1}\right)$, and their testes were exposed under the dissecting microscope. The left testis was treated as the experimental group and the right testis remained completely untouched throughout the procedure to act as a control. The accuracy of the injection was monitored by adding $0.04 \%$ Trypan blue to the DNA solution. The DNA solution was injected into the rete testis using a glass injection pipette visualized with a microscope. ${ }^{31}$ Injection into the rete testis was chosen over direct injection into the testis because it introduced the plasmid DNA directly into the seminiferous tubules. This method resulted in little damage to the testes. ${ }^{24,28}$ To increase the injection volume, the injection pipette was connected to a polyethylene tube filled with the DNA solution, and the injection was performed until almost all of the surface tubules (80\%-100\%) became stained with Trypan blue (typically $40-70 \mu \mathrm{l}, 25-50 \mu \mathrm{g}$ ). After the DNA injection, EP was performed with an Electro Square Porator ECM-2001 (BTX, San Diego, CA, USA). The testis was held in a tweezers-type electrode (BTX). Square electrical pulses were applied six times at $30-60 \mathrm{~V}$ with a time constant of 30-50 $\mu$ s following the technique of Muramatsu et al. ${ }^{23}$ The rats were subsequently sacrificed at 3, 5, 9, 13, 22 and 60 days $(n=4)$ after surgery. Testes (left experimental and right negative control) from the animals killed on specified days were torn apart in phosphate-buffered saline (PBS) and analyzed in vivo using bioluminescence imaging. During the imaging, two consecutive scans of 2 min each were acquired (excitation wavelength, $480 \mathrm{~nm}$; emission wavelength, $530 \mathrm{~nm}$ ). The captured images were quantified using the Living Image software package (Xenogen; Model Animal Research Institute, Nanjing University, China). The images were represented using a pseudocolour scale, with the colors red and blue representing the most and least intense luminescence, respectively.

To evaluate GFP expression in epididymis-matured sperm (with the exception of the sperm viability assay, the sperm motility was maintained at greater than or equal to $72 \%$ in all of the experiments, and the decrease rate of the sperm motility was kept to within $5 \%$ during handling), rats were killed, and their epididymides were separated from their testes. The testes were snap-frozen in liquid nitrogen for other analyses. The epididymal sperm were obtained by partially macerating the epididymis in PBS and allowing the sperm to swim freely from the surrounding tissue. The epididymal sperm were then analyzed using a BD FACSCalibur flow cytometer (Becton Dickinson, Franklin Lakes, NJ, USA) and Cell Quest software (Becton Dickinson).

\section{Detection of spermatogenic CatSper2 knockdown efficiency}

Quantitative polymerase chain reaction and Western blot analysis were performed to determine the efficiency of CatSper2 knockdown in the transfected testes and epididymal sperm samples collected from 3 to 60 days. Quantitative RT-PCR was performed with the SYBR PrimeScript RT-PCR Kit (TaKaRa, Dalian, China). Glyceraldehyde3-phosphate dehydrogenase was used in parallel for each run as an 
internal control. The 115-bp CatSper2 and 317-bp glyceraldehyde-3phosphate dehydrogenase products that spanned 3-5 exons were amplified using the following primers: 5'-GGCTTGAGCCAAGCTGTACCA- $3^{\prime}$ as the forward primer and $5^{\prime}$-AAGCGCACTAGCTGCTCCTGA-3' as the reverse primer for CatSper2, and $5^{\prime}$ ATCACTGCCACTCAGAAG-3' as the forward primer and $5^{\prime}$ AAGTCACAGGAGACAACC- $3^{\prime}$ as the reverse primer for glyceraldehyde-3-phosphate dehydrogenase. All of the primers were synthesized by Invitrogen (Shanghai, China). After protein quantification, the cell extract was resolved by $10 \%$ sodium dodecyl sulfate-polyacrylamide gel electrophoresis (SDS-PAGE) and was electroblotted onto nitrocellulose membranes (Bio-Rad, Shanghai, China). The membranes with the transferred proteins were incubated with goat anti-rat CatSper2 polyclonal antibody (1:800; Santa Cruz Biotechnology, Santa Cruz, CA, USA) as the primary antibody, followed by incubation with horseradish peroxidase-conjugated rabbit anti-goat immunoglobulin (IgG) $(1: 3000)$ as the secondary antibody. Chemiluminescence reaction was carried out with an ECL kit (Pierce, Rockford, IL, USA) for 1 min and then exposed to X-Omat radiographic film (Kodak, Shanghai, China). To assess sample loading, blots were stripped and reprobed using anti- $\beta$ actin $(1: 1000)$ and a goat anti-mouse IgG-HRP conjugate $(1: 3000)$. The amount of each protein sample was controlled by $\beta$-actin.

\section{Epididymal sperm intracellular $\mathrm{Ca}^{2+}$ determinations and image analysis}

Epididymal sperm cells $\left(5 \times 10^{6}\right.$ cells $\left.\mathrm{ml}^{-1}\right)$ were stained with $5 \mu \mathrm{mol}{ }^{-1}$ of fluo3-AM, a fluorescent $\mathrm{Ca}^{2+}$ indicator dye (in $0.5 \%$ DMSO) for $40 \mathrm{~min}$ at $37{ }^{\circ} \mathrm{C}$ in HEPES medium (containing $400 \mathrm{mmol} \mathrm{l}^{-1}$ sucrose, $50 \mathrm{mmol} \mathrm{l}^{-1} \mathrm{NaCl}, 5 \mathrm{mmol} \mathrm{l}^{-1} \mathrm{KCl}$, $20 \mathrm{mmol} \mathrm{l}^{-1}$ HEPES (pH 7.4), $1 \mathrm{mmol} \mathrm{l}^{-1}$ EGTA). ${ }^{32}$ Extracellular dye was removed by centrifugation at $170 \mathrm{~g}$ for $5 \mathrm{~min}$. Sperm were resuspended in HEPES medium and incubated for an additional $20 \mathrm{~min}$ to allow for de-esterification of the dye into its charged and $\mathrm{Ca}^{2+}$-sensitive form. Then, $0.5 \mathrm{ml}$ of the sperm suspension (loaded with fluo-3 AM) was transferred to a $3 \mathrm{ml}$ acrylic cuvette and held at a constant temperature of $37-39{ }^{\circ} \mathrm{C}$ with constant stirring A BD FACSCalibur flow cytometer running Cell Quest software was used to assess and analyze the data. Hyperactivation was then induced as previously described ${ }^{33}$ by incubating sperm in Tyrode's albumin lactate pyruvate solution containing $50 \mathrm{\mu mol}^{-1}$ thimerosal (containing EGTA) and in Bigger-Whitten-Whittinghan medium containing $5 \mathrm{mmol}^{-1}$ procaine. Response calibration was carried out by measuring the fluorescence intensity of the tetracarboxylate form of the indicator in solutions with precisely known free $\mathrm{Ca}^{2+}$ concentrations. Calibration solutions were based on EGTA $\mathrm{Ca}^{2+}$ HEPES buffering. The following equation was used to determine the ion dissociation constant $\left(K_{\mathrm{d}}\right)$ :

$$
\left[\mathrm{Ca}^{2+}\right]_{\text {free }}=K_{\mathrm{d}} \times\left(F-F_{\min }\right) /\left(F_{\max }-\mathrm{F}\right)
$$

where $F_{\min }$ was the fluorescence intensity of the indicator in the absence of calcium, $F_{\max }$ was the fluorescence of the calcium-saturated indicator and $F$ was the fluorescence at intermediate calcium levels. When the $K_{\mathrm{d}}$ was known, the same equation was used to obtain $\left[\mathrm{Ca}^{2+}\right]_{\text {free }}$ for experimental samples from the measured values of $F$. Fluorescence intensity was detected with a flow cytometer using a $480 \pm 40 \mathrm{~nm}$ excitation filter and a 535 $\pm 50 \mathrm{~nm}$ emission filter. A side angle (SSC) F1 (fluo-3 AM) plot was used to identify the sperm. An F1 histogram was used to mark the mean fluorescence intensity. In each treated group, 10000 sperm were detected. The ratio method by Grynkiewicz et al. ${ }^{34}$ was used to calculate the sperm cell $\mathrm{Ca}^{2+}$ concentration.
The fluorescence of individual, motile sperm in an open microchamber at $39{ }^{\circ} \mathrm{C}$ was monitored for single-cell $\mathrm{Ca}^{2+}$ imaging before and after the treatments. Fluorescence intensity was detected with an epifluorescence microscope using a $480 \pm 40 \mathrm{~nm}$ excitation filter and a $535 \pm 50 \mathrm{~nm}$ emission filter with an oil immersion $\times 40$ Fluar objective (Leica Inc., Oskar-Barnack-Straße, Germany).

\section{Fertilizing capacity of epididymal sperm after in vivo EP}

In order to compare the fertilizing capacity of spermatozoa with cauda epididymides, in vitro fertilization experiments were carried out with treated and control sperm. The procedure for in vitro fertilization was done as previously described. ${ }^{35,36}$ Briefly, immature female rats (250$300 \mathrm{~g}$ ) were given $600 \mathrm{IU}$ of pregnant mare's serum gonadotropin and 600 IU of human chorionic gonadotrop(h)in (Ningbo Second Hormone Co., Ltd, Beijing, China) and were killed 68-70 h later by pentobarbitone sodium. Their ovaries and oviducts were dissected in PBS, and the oviducts were ruptured by needle to release the cumulus mass. Diluted sperm suspension $\left(150 \mu \mathrm{l}, 7 \times 10^{5}\right.$ spermatozoa $\left.\mathrm{ml}^{-1}\right)$ was transferred to the $450 \mu \mathrm{l}$ in vitro fertilization dishes containing 5-10 oocytes (Fertilization Medium K-SIFM-20; Cook Medical, Brisbane Technology Park, Brisbane, Qld, Australia). In previous cases where the zona pellucida was removed, the ovulated mouse eggs were treated with hyaluronidase (100 units $\mathrm{ml}^{-1}$, type I-S; Sigma, Shanghai, China) to remove zona pellucida, and then the eggs were washed and exposed briefly to acidic Tyrode's solution to dissociate the zona pellucida. ${ }^{37}$ Gametes were cocultured at $37{ }^{\circ} \mathrm{C}$ under $5 \% \mathrm{CO}_{2}$ for 5 h (Cleavage Medium K-SICM-20; Cook Medical). Subsequently, all the oocytes and zygotes were transferred to a washing medium to remove excess spermatozoa. The oocytes and zygotes were examined 19-24 h after insemination as previously described. ${ }^{35,36,38}$ Successful fertilization of an oocyte was scored as two-cell embryos 25-28 h after insemination.

\section{Epididymal sperm cell motility and viscosity experiment assays} Spermatozoa were obtained by applying gentle pressure to excised caudal epididymal tissue nicked at four sites with scissors. Cells that dispersed into the modified Bigger-Whitten-Whittinghan solution at $37{ }^{\circ} \mathrm{C}$ were collected after $15 \mathrm{~min} .{ }^{18}$ Subsequently, sperm cells were observed on slides on a $37^{\circ} \mathrm{C}$ stage of a Nikon 35 microscope and were videotaped using $\times 400$ differential interference microscopy (Nikon Microscopy Inc., Tokyo, Japan) Stroboscopic illumination at $30 \mathrm{~Hz}$ was provided by a xenon flash tube (Chadwick-Helmuth Co., El Monte, CA, USA). Computer-assisted semen analysis (CASA) was performed to analyze the sperm by a $\times 4$ Nikon negative-phase objective. Video images were digitized (30 frames at $60 \mathrm{~Hz}$ ) and analyzed using HTM-IVOS, ver. 10 (Hamilton-Thorne Research, Beverly, MA, USA). Sperm motility was assessed by video capture of the swimming pattern and by CASA analysis (WLJY-9000) of path velocity (VAP), straight line velocity (VSL), curvilinear velocity (VCL), amplitude of lateral head displacement (ALH) and linearity (LIN). From each treated sample, $400-450$ motile sperm were analyzed. Each experiment was repeated three times using three males from each treatment. Because the movement of the sperm heads depended on flagellar bending and beating patterns, increased VCL and ALH, and decreased LIN were indicative of hyperactivation. ${ }^{39,40}$

Sperm cells that dispersed into $1 \mathrm{ml}$ prewarmed $\left(37^{\circ} \mathrm{C}\right)$ modified Bigger-Whitten-Whittinghan medium were assessed for viability using a live-to-dead count ratio. After the sperm were incubated for $3 \mathrm{~min}, 100 \mu \mathrm{l}$ of sperm were mixed with $9 \mathrm{mmol} \mathrm{l}^{-1} 5$-carboxyfluorescein diacetate (Sigma) and $24 \mu \mathrm{mol} \mathrm{l}^{-1}$ of the nuclear stain 
propidium iodide (Sigma). After a 5-min incubation, samples were analyzed using fluorescence microscopy. At least 200 sperm were counted per epididymis, and the number of live sperm versus dead sperm was determined. In the viscosity experiments, long-chain polyacrylamide (Invitrogen, Shanghai, China) was dissolved directly in the medium by stirring overnight at room temperature, resulting in a $2 \%$ stock solution (viscosity of $4000 \mathrm{cP}$, according to the manufacturer's instructions; $1 \mathrm{P}=0.1 \mathrm{~Pa}$ s). This medium was diluted to $0.75 \%$ with freshly prepared medium and $18 \mu$ of the medium was overlain onto $2 \mu \mathrm{l}$ of spermatozoa in medium on a prewarmed $80 \mu \mathrm{m}$ chamber slide. A coverslip was then placed over the sample, and an analysis was performed with the WLJY-9000 Sperm Analyzer, as described above. In the assay, means were obtained from the 20 sperm measured for each group (mock, negative control and treatment).

\section{Statistical analysis}

All of the data were obtained from one independent experiment carried out in triplicate and were presented as mean \pm s.d. The main and interactive effects were analyzed by a one-way ANOVA using SPSS13 software (SPSS Inc., Chicago, IL, USA). When justified by one-way ANOVA, differences between individual groups were analyzed by Fisher's PLSD test. Differences were considered statistically significant if $P<0.05$.

\section{RESULTS}

Transfection efficiency and cellular targets of plasmid DNA in the testis

The bioluminescence imaging signal data (Figure 1) from this study demonstrated that there was a strong positive correlation of the plasmid GFP signal with the amount of plasmid injected when the injection amount ranged from 25 to $45 \mu \mathrm{g}$. The testicular GFP signal was the
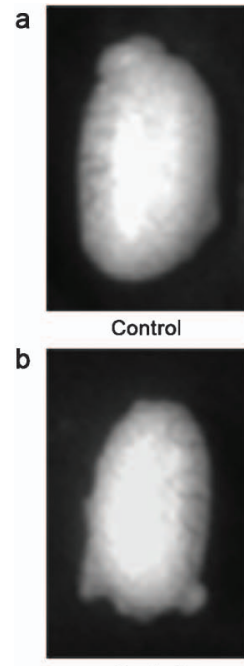

Control

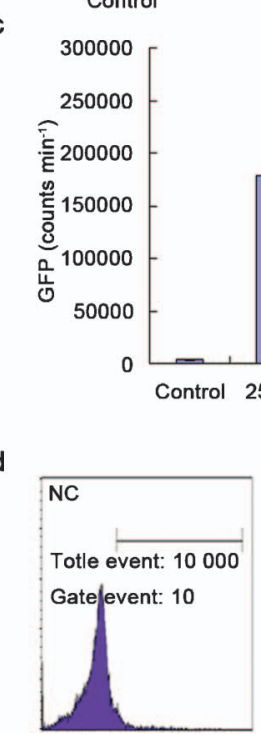

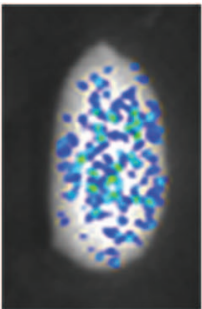

$25 \mu g$

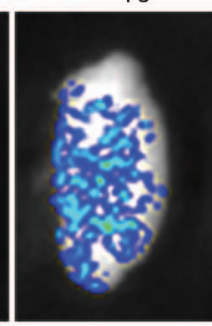

3 days

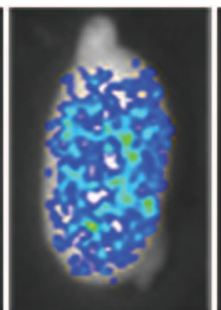

$30 \mu \mathrm{g}$

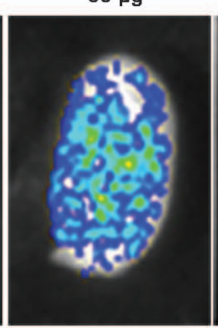

5 days

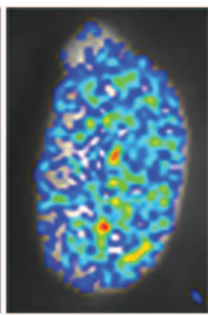

$35 \mu \mathrm{g}$

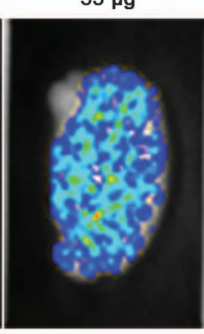

9 days

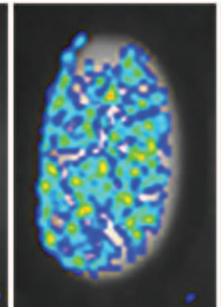

$40 \mu \mathrm{g}$

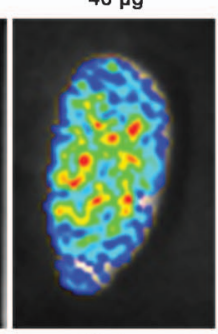

13 days

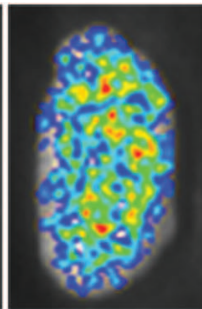

$45 \mu \mathrm{g}$

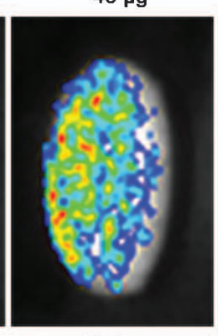

22 days

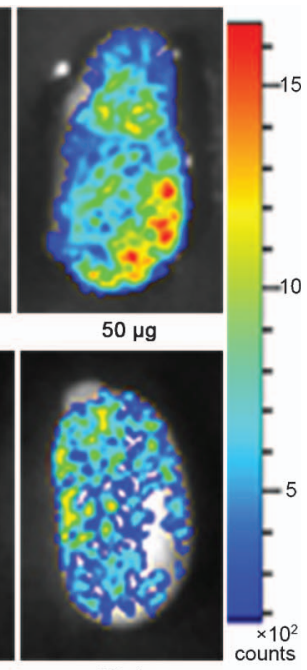

60 days

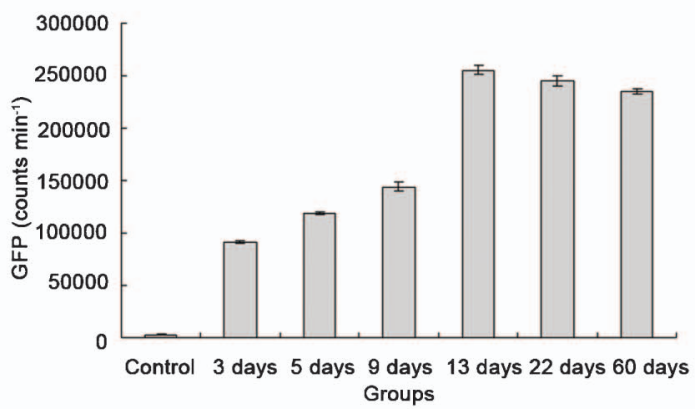

Groups
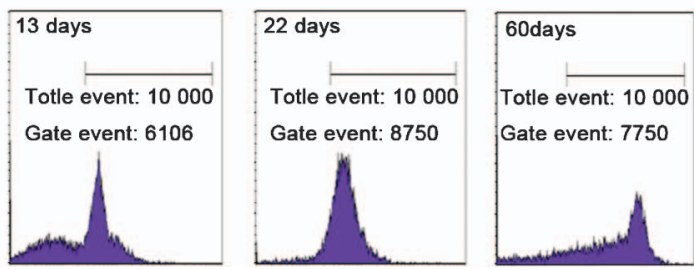

Figure 1 The detection of the sperm transfection rate using in vivo Imaging-200 System (IVIS-200 System) and flow cytometry. (a) The intensity of GFP expressed in rat testis as a function of the plasmid dose. (b) The intensity of GFP expressed in rat testis observed up to 60 days following plasmid transfection via in vivo testis electroporation. The GFP fluorescence intensity was detected by the IVIS-200 System (Xenogen; Caliper Life Sciences, Hopkinton, MA, USA). The pseudocolor image of the rat testis represents the relative expression of GFP. (c) The exactitude fluorescence intensity histogram description of $\mathbf{a}$ and $\mathbf{b}$ ( $n=3$ ). (d) The GFP-positive epididymal-derived sperm, as detected by flow cytometry: 0.7\% (704/10 000) GFP-positive sperm are detected at 5 days, while $32.7 \%$ (3274/10 000) and 61.1\% $(6106 / 10000)$ are shown at 9 days and 13 days, respectively, after transfection. The maximum percentage of positive cells is observed at 22 days, which is $87.5 \%$ (8750/10 000), and 77.5\% (7750/10 000) of positive cells are found at 60 days in rat epididymis $(n=3)$. GFP, green fluorescent protein; NC, negative control. 
strongest when each side of the testicle was injected with $70 \mu \mathrm{l}$ PBS solution containing $50 \mu \mathrm{g}$ pGP/U6/GFP/Neo-siRNA-NC. The signal was weaker when the $70 \mu \mathrm{l}$ PBS contained $45 \mu \mathrm{g}$ pGP/U6/GFP/Neo-siRNA-NC, but the difference between the two signals was not statistically significant. In the 60-day test period, the testicular fluorescence signal peaked at 13 days and diminished thereafter; however, the strong fluorescence signal could still be detected on 60 days and showed no statistical difference from the signal on 13 days.

The flow cytometric analysis of epididymal sperm from the initial transfection (Figure 1d) showed that few cells with a fluorescence signal were detected. As the expression of shRNA in the testis increased, the number of GFP-positive sperm cells in the epididymis increased to $87.5 \% 22$ days after transfection and decreased to $77.5 \%$ by 60 days, but no significant difference was found between the two numbers of GFP-positive sperm.
The sperm morphology test was not repeated in this experiment because the sperm morphological analysis in our previous in vitro EP-mediated siRNA transfection experiments confirmed that both sperm morphology and physiology were not severely affected by EP. ${ }^{30}$

As shown in Figure 2a, the knockdown effect of shRNA specific to CatSper2 mRNA in testis and epididymal sperm was markedly timedependent. The maximum knockdown to testicular CatSper 2 mRNA reached $88.0 \% 13$ days after transfection and decreased afterwards. However, the knockdown was still at $70.1 \%$ at 60 days, a value not much different from the value at 13 days, which indicated a long period of effective knockdown. The CatSper2 mRNA knockdown in epididymal sperm reached the peak value at 22 days, later than in the testis. The CatSper2 mRNA knockdown was $83.4 \%$ at 22 days after transfection in vivo, and it decreased very little afterwards.
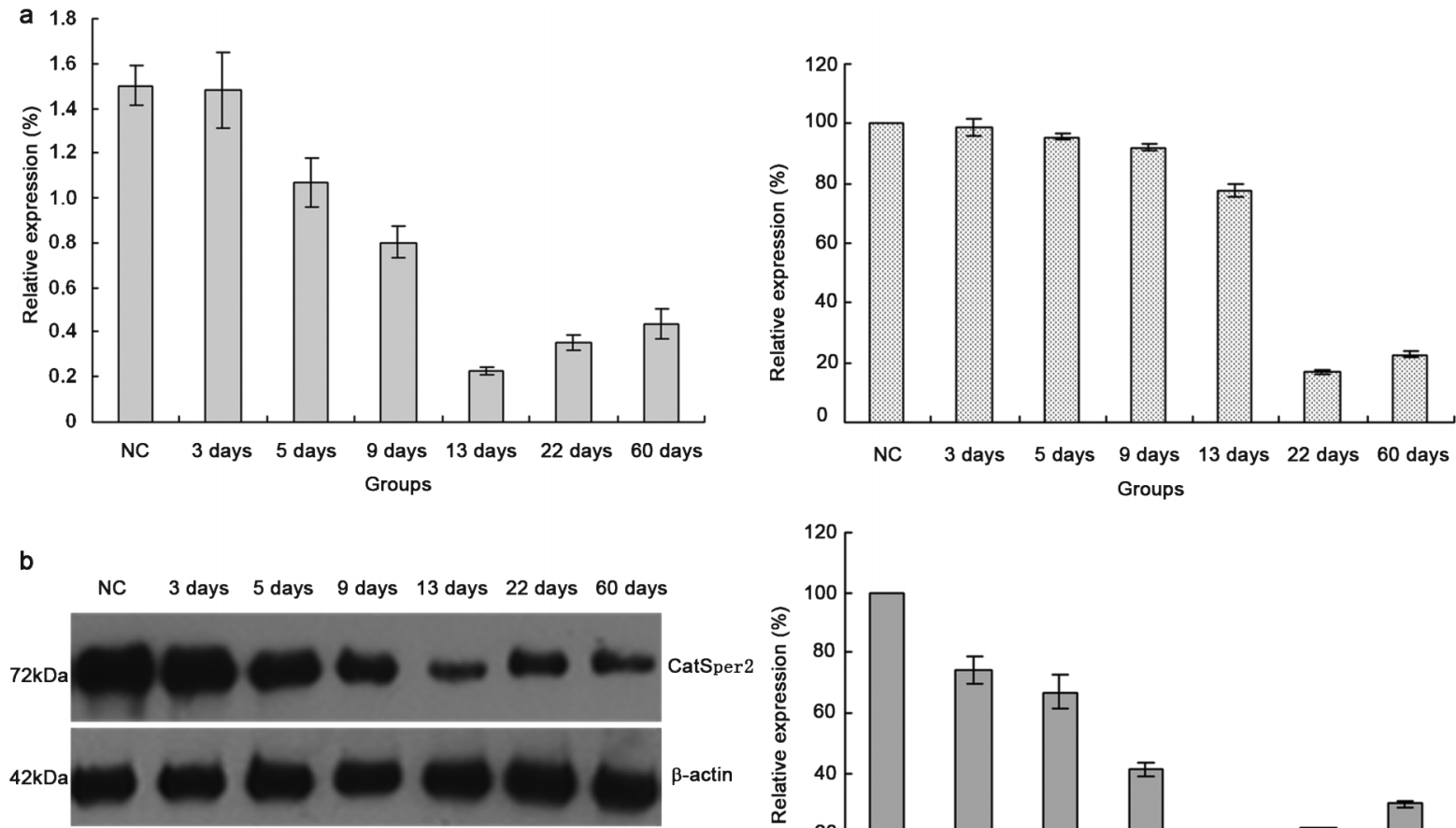

Rat testes

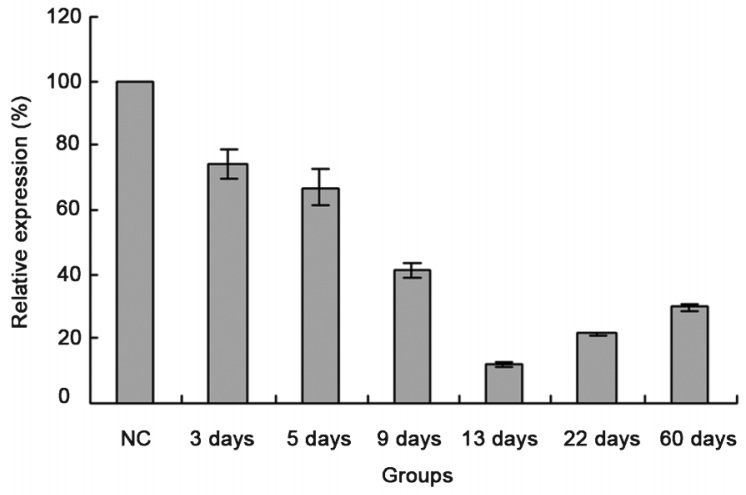

C
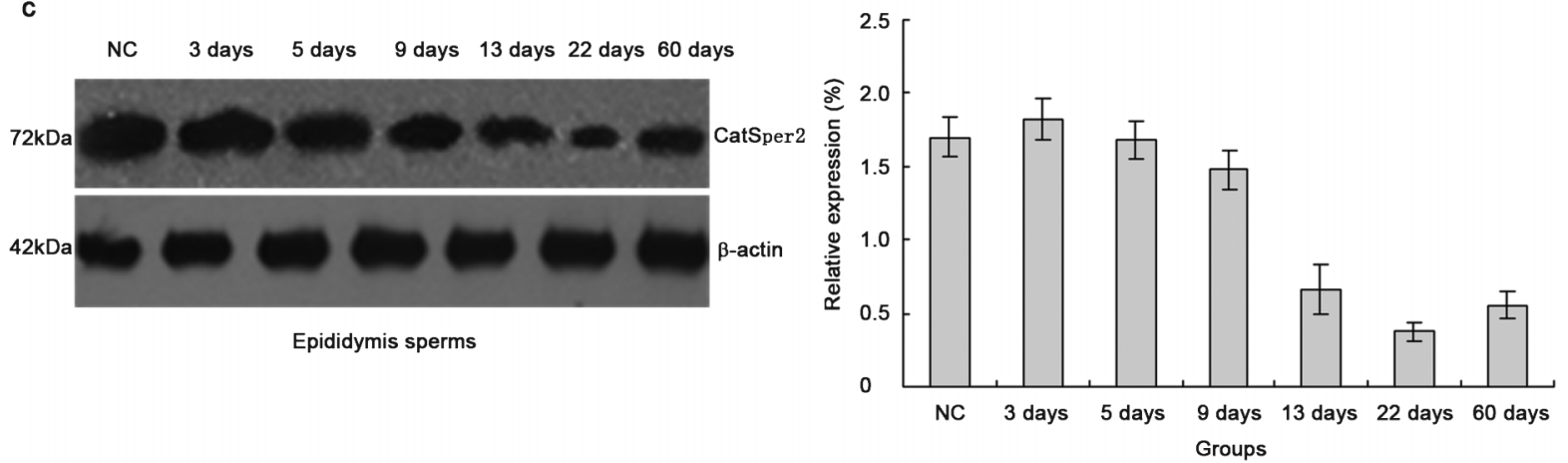

Figure 2 Knockdown efficiency of plasmid on CatSper2 ion channel proteins in testis and epididymis. (a) The relative expression of CatSper2 mRNA in testis (left) and epididymal sperms (right). (b) The knockdown of CatSper2 protein in testis. The Western blot analysis of shRNA-CatSper2 knockdown in testis (left) and the exactitude histogram description of relative expression of CatSper2 after interference in testis (right). (c) The knockdown of CatSper2 protein in epididymal sperm. The Western blot analysis of shRNA-CatSper2 knockdown in epididymal sperm (left) and the exactitude histogram description of relative expression of CatSper2 after interference in epididymal sperm (right). NC, negative control; shRNA, short hairpin RNA. 
The anti-rat CatSper2 antibody Western blot analysis showed that shRNA significantly blocked Catsper2 protein expression in testis and epididymal sperm in a strongly time-dependent manner. Figure $\mathbf{2 b}$ and $\mathrm{c}$ shows that, after electrotransfection in vivo, the highest knockdown appeared in testicular tissue $(84.87 \%)$ on 13 days and in epididymal sperm (77.89\%) on 22 days. With shRNA transcribed in testis, the relative expression of Catsper 2 in testis and epididymal sperm did not appear to recover within a 60-day period. The knockdown of CatSper2 proteins in testis and epididymis was still at $70.87 \%$ and $67.49 \%$, respectively, at the end of the 60 -day test.

\section{cAMP-induced $\mathrm{Ca}^{2+}$ influx and hyperactivation in epididymal sperm}

As shown in Table 1, the intracellular $\mathrm{Ca}^{2+}$ concentration of the treated and control sperm increased markedly when sperm were incubated in the Tyrode's albumin lactate pyruvate medium containing thimerosal, independent of the presence of extracellular $\mathrm{Ca}^{2+}$ in the medium. Meanwhile, fluorescent imaging of fluo3-loaded individual sperm demonstrated that thimerosal raised the $\mathrm{Ca}^{2+}$ concentration in the head and flagellum within $25 \mathrm{~s}$ (Figure 3). However, the high concentration was maintained for only a very short time without supplementing with extracellular $\mathrm{Ca}^{2+}$. In contrast, a high intracellular $\mathrm{Ca}^{2+}$ concentration also appeared in control groups when the sperm were incubated in procaine with a $5 \mathrm{mmol}^{-1}$ final concentration for $4 \mathrm{~h}$. The $\mathrm{Ca}^{2+}$ concentration of the treated groups was higher than that in the normal group, but no significant difference was seen between the two groups (Table 1). The $\mathrm{Ca}^{2+}$ concentration of the treated groups was far below the normal concentration of 200$1000 \mathrm{nmol}^{-1}$ required for triggering hyperactivation.

Sperm were incubated for $1 \mathrm{~min}$ in Tyrode's albumin lactate pyruvate with $50 \mu \mathrm{mol} \mathrm{l}^{-1}$ of $\mathrm{Ca}^{2+}$-free thimerosal (containing EGTA). Critical velocity or track speed (VCL), amplitude of lateral head displacement (ALH) and beat cross-frequency (BCF) detected by CASA are listed in Table 2. The results indicated that more than $90 \%$ of the sperm were hyperactivated in both the control and treated groups. This was much higher than in the normal sperm, whose hyperactivation rate was only $5 \%$ $10 \%$. Subsequently, the sperm were incubated for $5 \mathrm{~min}$ in capacitation solution with procaine at a final concentration of $5 \mathrm{mmol}^{-1}$ CASA analysis indicated that the hyperactivation rate of treated sperm dropped to $11.1 \%$. The swimming pattern of the sperm was characterized by helical motility with narrow amplitude and high frequency of flagellar beating, as previously reported. ${ }^{13}$ However, the control group maintained a high hyperactivation rate of $95.7 \%(P<0.05)$ (Table 2$)$ for a longer time when no blocking agents were added.

Fertilization efficiency suppressed by CatSper2 expression decline The feasibility of the CatSper protein family being a target for male contraception depends on the fertilizing capacity of the sperm after RNA interference. In this study, mature sperm from rat cauda

Table 1 Flow cytometer (FCM) detection of sperm intracellular $\left[\mathrm{Ca}^{2+}\right]$

\begin{tabular}{|c|c|c|c|c|}
\hline \multirow[t]{2}{*}{ Groups } & \multicolumn{2}{|c|}{ Control $\left(\mathrm{nmol}^{-1}\right)$} & \multicolumn{2}{|c|}{ SiRNA-785 (nmol $\left.\Gamma^{-1}\right)$} \\
\hline & $\mathrm{Oh}$ & $4 \mathrm{~h}$ & $\mathrm{Oh}$ & $4 \mathrm{~h}$ \\
\hline Capacitation & $42.6 \pm 0.8^{a}$ & $56.6 \pm 0.5^{a}$ & $42.5 \pm 0.4^{a}$ & $41.1 \pm 1.1^{\mathrm{a}}$ \\
\hline Thimerosal & $235.1 \pm 3.8^{b}$ & $62.7 \pm 0.7^{a}$ & $232.0 \pm 3.2^{b}$ & $57.3 \pm 0.9^{b}$ \\
\hline Procaine & $241.8 \pm 1.7^{b}$ & $216.7 \pm 1.7^{b}$ & $57.2 \pm 1.1^{\mathrm{c}}$ & $61.5 \pm 0.8^{b}$ \\
\hline
\end{tabular}

The results are presented as means \pm s.d. The different small letters in the same column indicate significant difference $(P<0.05)$.

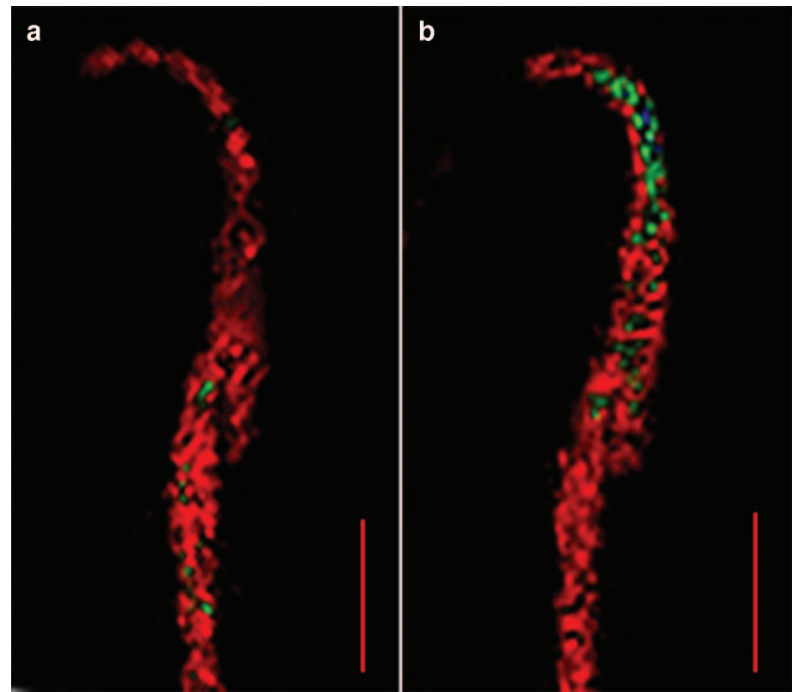

Figure $3 \mathrm{Ca}^{2+}$ imaging of fluo3-loaded sperms. Pictures were taken within $25 \mathrm{~s}$ of applying medium without thimerosal (a) and with thimerosal (solution containing EGTA) (b). The green spots indicated higher fluorescent intensities and increased intracellular $\mathrm{Ca}^{2+}$ (scale bars $=10 \mu \mathrm{m}$ ).

epididymis were collected and used to fertilize eggs with an intact zona pellucida in vitro. After 19-24 h of fertilization cleavage, no significant difference was shown in the sperm fertilization rate among the treated groups (from 0 to 13 days, $45.4 \%, n=10 / 22$ ), the NC group $(46.7 \%$, $n=14 / 30)$ and the normal group $(45.8 \%, n=11 / 24)$ (Figure 4a). With the increased transcription of shRNA in vivo and the constant renewal of sperm in the epididymis, only $12.5 \%(n=4 / 32)$ of oocytes were fertilized by epididymal sperm from the 22 days treated group, evidently lower than $46.4 \%(n=13 / 28)$ from the NC group and $45.8 \%$ $(n=11 / 24)$ from the normal group. However, when the sperm from rat cauda epididymis fertilized eggs with the extracellular matrix removed, no significant difference in cleavage rate was shown among the three groups $(P>0.05)$.

Viability and motility of transfected sperm in viscoelastic solution Sperm quality was further investigated. The sperm cells were assessed for viability using a live-to-dead count ratio with propidium iodide staining. Compared with the control, the treated groups showed differences in fertilizability, hyperactivation rates and intracellular $\mathrm{Ca}^{2+}$ peak, but no differences were seen in the survival rate (Figure 5). To further reveal the motility status of sperm with low CatSper2 expression, a migration assay was performed in viscoelastic solution. As shown in Figure 5, in normal cell medium containing $0.75 \%(w / v)$ long-chain polyacrylamide, the treated spermatozoa were immobilized, whereas the spermatozoa of the control and normal groups remained progressively mobile, albeit with their forward velocity reduced by about $50 \%$.

\section{DISCUSSION}

The CatSper protein family plays an important role in sperm hyperactivation and fertilizing capacity. ${ }^{14}$ For the spermatozoa, abnormal expression of the CatSper protein subunit will not only lead to failure in acquiring hyperactivation, but also in the capability of penetrating the zona pellucida. In this study, CatSper2 expression was suppressed by microinjection of plasmid DNA into the rete testis combined with $\mathrm{EP}$, which indicated that male fertility could be manipulated by blocking CatSper2 expression in order to suppress the signal channel of sperm hyperactivation. Furthermore, microinjection of EP-mediated 
Table 2 CASA analysis of the treated and control sperm

\begin{tabular}{|c|c|c|c|c|}
\hline Groups & & The non-treated groups & The thimerosal-treated groups & The procaine-treated groups \\
\hline \multirow[t]{2}{*}{ Motile (\%) } & Control & $65.4 \pm 0.9^{a}$ & $66.8 \pm 1.0^{\mathrm{a}}$ & $63.5 \pm 0.4^{b}$ \\
\hline & siRNA-785 & $64.3 \pm 0.7^{\mathrm{a}}$ & $60.6 \pm 0.4^{c}$ & $57.6 \pm 0.4^{d}$ \\
\hline \multirow[t]{2}{*}{$\operatorname{VCL}\left(\mu \mathrm{mol} \mathrm{I}^{-1} \mathrm{~s}^{-1}\right)$} & Control & $303.6 \pm 1.8^{\mathrm{a}}$ & $345.3 \pm 1.4^{b}$ & $362.1 \pm 0.5^{c}$ \\
\hline & siRNA-785 & $210.4 \pm 3.1^{\mathrm{d}}$ & $285.3 \pm 2.8^{\mathrm{e}}$ & $212.5 \pm 2.9^{d}$ \\
\hline \multirow[t]{2}{*}{$\mathrm{ALH}\left(\mu \mathrm{mol} \mathrm{I}^{-1}\right)$} & Control & $17.3 \pm 0.6^{\mathrm{a}}$ & $20.8 \pm 0.9^{b}$ & $19.1 \pm 0.4^{\mathrm{b}}$ \\
\hline & SiRNA-785 & $12.2 \pm 0.6^{c}$ & $20.7 \pm 0.4^{b}$ & $11.3 \pm 0.4^{c}$ \\
\hline \multirow[t]{2}{*}{$\mathrm{BCF}(\mathrm{Hz})$} & Control & $19.7 \pm 0.3^{\mathrm{a}}$ & $13.9 \pm 0.3^{b}$ & $23.6 \pm 0.3^{c}$ \\
\hline & siRNA-785 & $25.6 \pm 0.3^{d}$ & $14.8 \pm 0.2^{b}$ & $28.8 \pm 0.2^{\mathrm{e}}$ \\
\hline \multirow[t]{2}{*}{ Hyperactivation (\%) } & Control & $5.5 \pm 0.5^{\mathrm{a}}$ & $99.2 \pm 0.2^{\mathrm{b}}$ & $95.7 \pm 0.6^{\mathrm{b}}$ \\
\hline & siRNA-785 & $5.0 \pm 0.1^{a}$ & $97.5 \pm 0.6^{b}$ & $11.1 \pm 0.5^{c}$ \\
\hline
\end{tabular}

The results were represented as mean \pm s.e. The different small letters in the same parameter of different treatment method between the treated and control groups indicated marked difference $(P<0.05)$.

Abbreviations: ALH, amplitude of lateral head displacement; BCF, beat cross frequency; CASA, computer-assisted semen analysis; VCL, curvilinear velocity.

plasmid DNA did not affect the function of testis and the survival rate of sperm.

In non-hormonal or genetic contraceptive research, the testis is a good target because cellular renewal and biochemical reaction of spermatogenesis takes place in the seminiferous epithelium and results in the daily production of 100-200 million spermatozoa. ${ }^{23,29}$ Potential
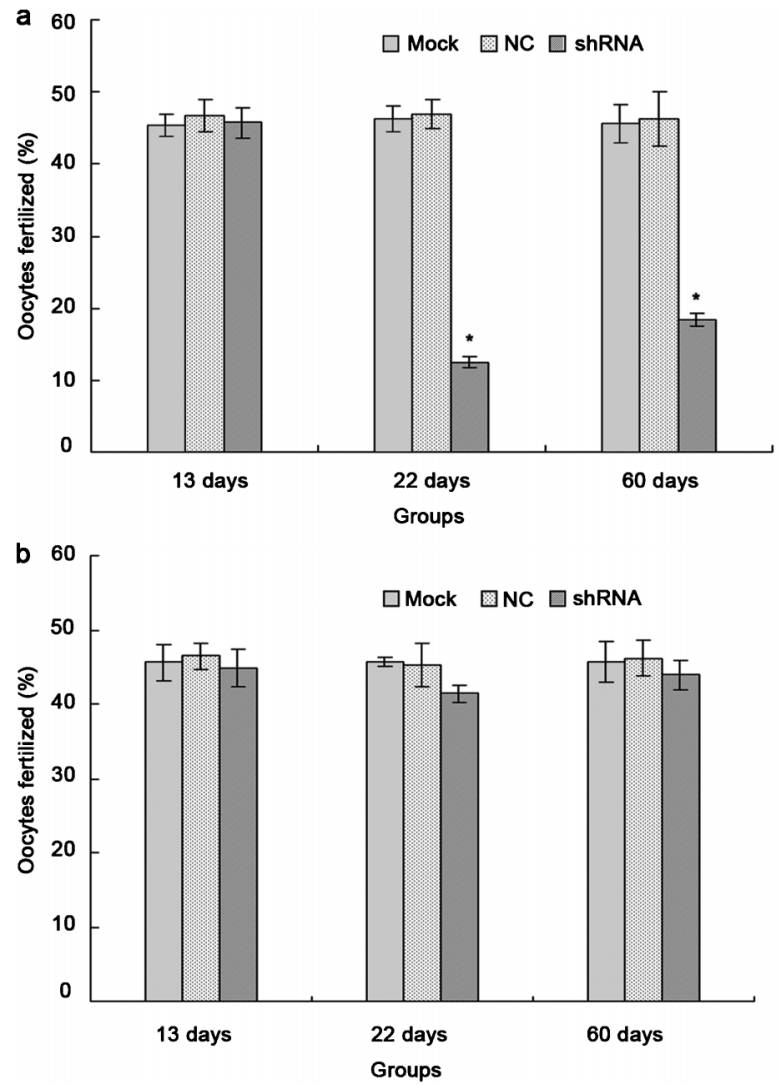

Figure 4 Fertilizing capacity of rat epididymal sperm after electrotransfection in vivo. The motility of sperm before and after treatment was unchanged (72\%). (a) The oocyte fertilization rate of rat sperm following interference in vivo, using unaltered mature oocytes. The fertilization capacity of treated sperm decreased compared to the control group at 22 and 60 days $(* P<0.05)$. (b) The oocyte fertilization rate of rat sperm following interference in vivo, using oocytes without zona pellucida $(n=3)$. There are no marked differences in the cleavage rate between the control group and the experimental group. NC, negative control; shRNA, short hairpin RNA. targets for fertility intervention might be identified by elucidating the mechanisms of the events of spermatogenesis. In vivo gene transfer into the testis by EP has been put forward as a non-hormonal approach to study the testis and sperm function, and as a novel way of creating transgenic animals. ${ }^{41-43}$ Selective inhibitors of ion channels could, in principle, inhibit sperm function and prevent fertilization. By applying the patch-clamp technique to mature human spermatozoa, Lishko et al. ${ }^{44}$ and Strunker et al. ${ }^{45}$ independently found that the Catsper ion channel of human spermatozoa is synergistically activated by elevation
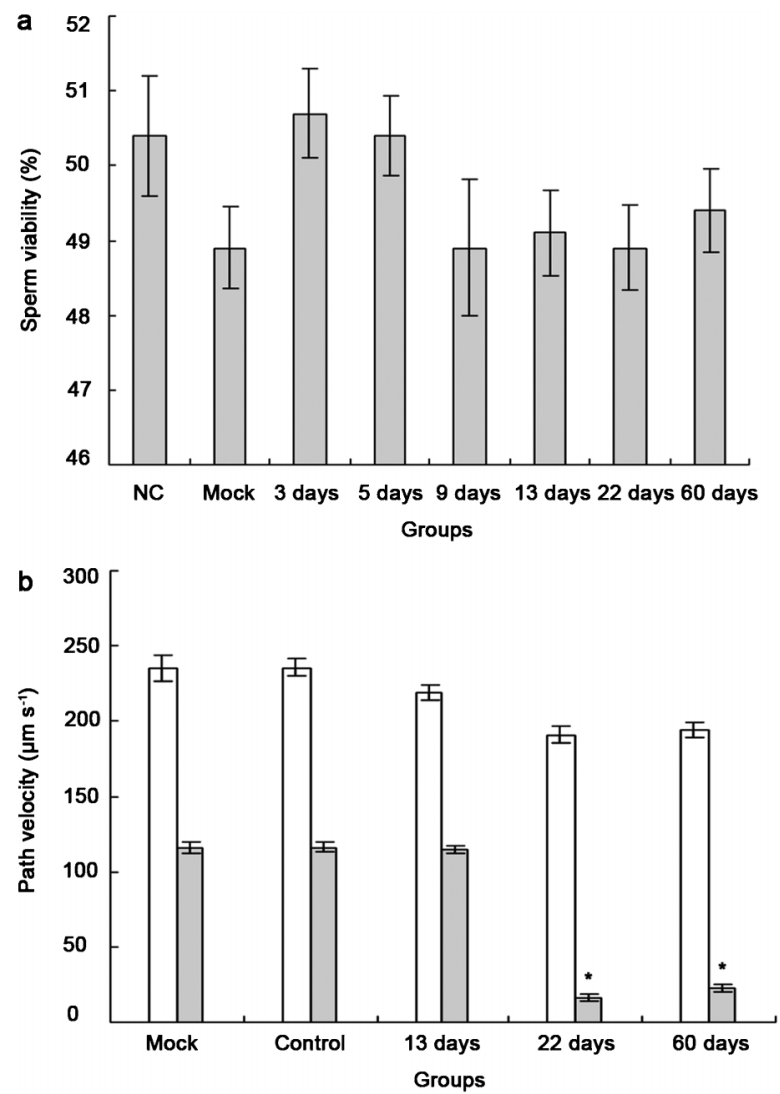

Figure 5 Viability and motility of transfected sperm in viscoelastic solution. (a) Sperm viability of the treated and control groups at different time points after transfection. (b) The open bars represent normal medium and the filled bars represent medium containing $0.75 \%(w / v)$ long chain polyacrylamide $(* P<0.05)$. NC, negative control. 
of the intracellular $\mathrm{pH}$ and extracellular progesterone, which is different from the genomic progesterone receptor. The CatSper ion channels represent a promising target for the development of a new class of non-hormonal contraceptives. ${ }^{46}$ Nifedipine, a commonly prescribed $\mathrm{Ca}^{2+}$ channel blocker in the treatment of high blood pressure and migraine, was found to have reversible contraceptive effects. ${ }^{25,47}$ In this study, we successfully blocked fertility in rats by knocking down CatSper2 ion channels with the help of EP-mediated plasmid DNA.

For sperm hyperactivation, a relatively high concentration of cellular $\mathrm{Ca}^{2+}$ is essential and must be sustained. ${ }^{48} \mathrm{Ca}^{2+}$ not only plays a role in motility but is also a key regulator in the initiation and maintenance of sperm hyperactivation. ${ }^{49,50}$ Our study showed that thimerosal induced hyperactivation in more than $90 \%$ of sperm (Table 2) because there was sufficient intracellular $\mathrm{Ca}^{2+}$ to initiate the hyperactivation, even without extracellular $\mathrm{Ca}^{2+}$. This result was similar to that seen by Ho and Suarez. ${ }^{33}$

Intracellular $\mathrm{Ca}^{2+}$ initiates sperm hyperactivation, but extracellular $\mathrm{Ca}^{2+}$ sustains hyperactivation. The availability of extracellular $\mathrm{Ca}^{2+}$ can be achieved by using procaine as an inducer. ${ }^{48}$ It was reported that, when induced by procaine, sperm almost immediately reverted to activated motility upon transfer into medium in which extracellular $\mathrm{Ca}^{2+}$ was buffered below $50 \mathrm{nmol} 1^{-1} \cdot{ }^{49,51}$ In this study, the control sperm acquired high hyperactivation motility when incubated in $5 \mathrm{mM}$ procaine for $5 \mathrm{~min}$, while the treated sperm did not (Table 2). The hyperactivation $(11.1 \%)$ of the treated sperm was higher than in the normal sperm $(5 \%-10 \%)$ because we did not completely block the expression of CatSper2 protein. However, the concentration of the cytoplasmic $\mathrm{Ca}^{2+}$ still remained low and could not evoke hyperactivation in treated sperm. Procaine failed to induce hyperactivation of treated sperm, which supported the statement that procaine evokes sperm hyperactivation by triggering $\mathrm{Ca}^{2+}$ influx, which is mediated by the $\mathrm{Ca}^{2+}$ channel on the cytolemma. ${ }^{40,52}$

In one mouse study, treated sperm showed relatively poor motility and progressive path velocity, but they had the same survival time as control sperm. The changes to flagellar beat increased the velocity of spermatozoa (path and track) and the frequency of abrupt turning. ${ }^{48}$ In this rat study, the results of CASA and confocal microscopic analysis indicated that both the forward velocity parameters (Table 1) and percentage of motility (Table 2) were slightly decreased in normal sperm and treated spermatozoa. However, the treated sperm significantly increased their flagellar beat. The experiment on the viability of sperm (Figure 5) showed no difference between the normal, control and treated groups. These results may also support the observation that although the treated sperm failed to fertilize the eggs with integrated zona pellucida, they did fertilize the naked eggs lacking zona pellucida. It has been suggested that CatSper2 is a unique cation channel protein required for normal sperm motility and particularly for sperm penetration of the zona pellucida. ${ }^{13}$

Removal of the egg zona pellucida enabled treated spermatozoa to fertilize the egg, suggesting that the zona pellucida of the egg represented an absolute barrier to spermatozoa lacking CatSper2. ${ }^{18}$ Compared to the untreated group, we found that treated spermatozoa failed to penetrate the zona pellucida of intact eggs (Figure 4). This finding suggests that, despite normal forward velocity and percentage of motile cells, the treated spermatozoa lack the power to penetrate the zona pellucida. To further verify the change of fertilization capability of the treated sperm, we tested the effects of elevating the viscosity of the medium on the motility of control and treated spermatozoa. We found that, although the mobility of all of the sperm was reduced in the higher viscosity media, the treated group showed a total loss of forward mobility, while the sperm of the control group still possessed $48.8 \%$ mobility (Figure $5 \mathbf{b}$ ). This result was consistent with previous research. ${ }^{53}$ CatSper2 was essential for generation of the hyperactivated form of motility, which was necessary for successful fertilization. ${ }^{13,18}$

Owing to a concern that male contraception induced by blocking CatSper2 could produce side effects, we observed the function of testis tissue and the duration of sperm survival. The results showed that microinjection of DNA plasmid and EP did not cause significant impact on the spermatogenesis cycle and testicular function (data not shown). Propidium iodide analysis indicated that the viability of the treated sperm was similar to that of the control sperm (Figure 5a).

In conclusion, this study showed that CatSper2 knockdown by EPmediated plasmid DNA could effectively interfere in male fertility, thus providing a prospective candidate for contraceptive drug development. However, plasmid DNA-mediated RNAi could not completely block protein expression as well as traditional gene knockout technology does. Furthermore, in vivo electroporation could only be realized through complicated surgery, which might influence the effect of blocking. Therefore, further study is necessary.

\section{AUTHOR CONTRIBUTIONS}

ZZ conceived and designed of the study, participating in whole experiment and draft the manuscript. GLW conceived of the study, coordinated the experiments performed by the members of the research team and helped to draft the manuscript. HXL and LL participated in the design of the study and in vivo electroporation. QWC and CBW performed the statistical analysis. FZ participated in the IVIS assay and helped with the statistical analysis.

\section{COMPETING FINANCIAL INTERESTS}

The authors declare that they do not have any competing financial interests.

\section{ACKNOWLEDGMENTS}

This work was supported by the National Key Technology R\&D Program (No. 2006BAD04A12) and Jiangsu Province Key Technology R\&D Program, China (No. BE2008306-2). The authors thank Professor Fei-Rong Gu and MSc Xuan Zhou of the college of Foreign Studies NJAU for their help in preparing the manuscript.

1 Anderson RA, Baird DT. Male contraception. Endocr Rev 2002; 23: 735-62.

2 Kamischke A, Nieschlag E. Progress towards hormonal male contraception. Trends Pharmacol Sci 2004; 25: 49-57.

3 WHO Task Force on Methods for the Regulation of Male Fertility. Contraceptive efficacy of testosterone-induced azoospermia in normal men. Lancet 1990; 336: 955-9.

4 Lopez LM, Grimes DA, Schulz KF. Nonhormonal drugs for contraception in men: a systematic review. Obstetr Gynecol Surv 2005; 60: 746-52.

5 Delves PJ, Lund T, Roitt IM. Antifertility vaccines. Trends Immunol 2002; 23: 213-9.

6 Jones AR, Cooper TG. A re-appraisal of the post-testicular action and toxicity of chlorinated antifertility compounds. Int J Androl 1999; 22: 130-8.

7 Dan J. Studies on the acrosome. III. Effect of calcium deficiency. Biol Bull 1954; 107: 335-49.

8 Bredderman PJ, Foote RH, Hansel W. The effect of calcium ions on cell volume and motility of bovine spermatozoa. Proc Soc Exp Biol Med 1971; 137: 1440-3.

9 Yanagimachi R, Usui N. Calcium dependence of the acrosome reaction and activation of guinea pig spermatozoa. Exp Cell Res 1974; 89: 161-74.

10 Young LG, Nelson L. Calcium ions and control of the motility of sea urchin spermatozoa. J Reprod Fertil 1974; 41: 371-8.

11 Ren D, Navarro B, Perez G, Jackson AC, Hsu S et al. A sperm ion channel required for sperm motility and male fertility. Nature $2001 ; 413$ : 603-9.

12 Shi YL, Bai JP, Wang WP. Ion-channels in human sperm membrane and contraceptive mechanisms of male antifertility compounds derived from Chinese traditional medicine. Acta Pharmacol Sin2003; 24: 22-30. 
13 Quill TA, Sugden SA, Rossi KL, Doolittle LK, Hammer RE et al. Hyperactivated sperm motility driven by CatSper2 is required for fertilization. Proc Natl Acad Sci USA2003, 100: 14869-74

14 Qi H, Moran MM, Navarro B, Chong JA, Krapivinsky G et al. All four CatSper ion channel proteins are required for male fertility and sperm cell hyperactivated motility. Proc Natl Acad Sci USA 2007; 104: 1219-23.

15 Carlson AE, Westenbroek RE, Quill T, Ren D, Clapham DE et al. CatSper 1 required for evoked $\mathrm{Ca}^{2+}$ entry and control of flagellar function in sperm. Proc Natl Acad Sci USA 2003; 100: 14864-8.

16 Quill TA, Ren D, Clapham DE, Garbers DL. A voltage-gated ion channel expressed specifically in spermatozoa. Proc Natl Acad Sci USA 2001; 98: 12527-31.

17 Jin JL, O'Doherty AM, Wang S, Zheng H, Sanders KM et al. Catsper3 and Catsper4 encode two cation channel-like proteins exclusively expressed in the testis. Biol Reprod 2005; 73: 1235-42.

18 Marquez B, Ignotz G, Suarez SS. Contributions of extracellular and intracellular $\mathrm{Ca}^{2+}$ to regulation of sperm motility: release of intracellular stores can hyperactivate CatSper1 and CatSper2 null sperm. Dev Biol 2007; 303: 214-21.

19 Li HG, Liao AH, Ding XF, Zhou H, Xiong CL. The expression and significance of CATSPER 1 in human testis and ejaculated spermatozoa. Asian J Androl 2006; 8 301-6.

20 Neumann E, Schaefer-Ridder M, Wang Y, Hofschneider PH. Gene transfer into mouse lyoma cells by electroporation in high electric fields. EMBO J 1982; 1: 841-5.

21 Brunner S, Furtbauer E, Sauer T, Kursa M, Wagner E. Overcoming the nuclear barrier Cell cycle independent nonviral gene transfer with linear polyethylenimine o electroporation. Mol Ther 2002; 5: 80-6.

22 St George JA. Gene therapy progress and prospects: adenoviral vectors. Gene The 2003; 10: 1135-41.

23 Muramatsu T, Shibata O, Ryoki S, Ohmori Y, Okumura J. Foreign gene expression in the mouse testis by localized in vivo gene transfer. Biochem Biophys Res Commun 1997: 233: 45-9.

24 Yamazaki $\mathrm{Y}$, Fujimoto $\mathrm{H}$, Ando $\mathrm{H}$, Ohyama $\mathrm{T}$, Hirota $\mathrm{Y}$ et al. In vivo gene transfer to mouse spermatogenic cells by deoxyribonucleic acid injection into seminiferous tubules and subsequent electroporation. Biol Reprod 1998; 59: 1439-44.

25 Huang Z, Tamura M, Sakurai T, Chuma S, Saito T et al. In vivotransfection of testicula germ cells and transgenesis by using the mitochondrially localized jellyfish fluorescent protein gene. FEBS Lett 2000; 487: 248-51.

26 Yamazaki Y, Yagi T, Ozaki T, Imoto K. In vivo gene transfer to mouse spermatogenic cells using green fluorescent protein as a marker. J Exp Zool 2000; 286: 212-8.

27 Ike A, Ohta H, Onishi M, Iguchi N, Nishimune $\mathrm{Y}$ et al. Transient expression analysis of the mouse ornithine decarboxylase antizyme haploid-specific promoter using in vivo electroporation. FEBS Lett 2004; 559: 159-64.

28 Kubota H, Hayashi Y, Kubota Y, Coward K, Parrington J. Comparison of two methods of in vivo gene transfer by electroporation. Fertil Steril 2005; 83: 1310-8.

29 Mruk DD. New perspectives in non-hormonal male contraception. Trends Endocrinol Metab 2008; 19: 57-64.

30 Zhang Z, Zhou X, Li HX, Cui QW, Yu J et al. Delivery of CatSper2 siRNA into rats sperms by electroporation repressed $\mathrm{Ca}^{2+}$ influx during sperm hyperactivation. Agric Sci China (Engl) 2011; 10: 101-5.

31 Brinster RL, Avarbock MR. Germline transmission of donor haplotype following spermatogonial transplantation. Proc Natl Acad Sci USA 1994; 91: 11303-7.

32 Bers DM, Patton CW, Nuccitellid R. A practical guide to the preparation of $\mathrm{Ca}^{2+}$ buffers. Methods Cell Biol 1994; 40: 3-29.
$33 \mathrm{Ho} \mathrm{HC}$, Suarez SS. An inositol 1,4,5-trisphosphate receptor-gated intracellular $\mathrm{Ca}^{2+}$ store is involved in regulating sperm hyperactivated motility. Biol Reprod 2001; 65 : 1606-15.

34 Grynkiewicz G, Poenie M, Tsien RY. A new generation of $\mathrm{Ca}^{2+}$ indicators with greatly improved fluorescence properties. J Biol Chem 1985; 260: 3440-50.

35 Holloway AJ, Moore HD, Foster PM. The use of rat in vitro fertilization to detect reductions in the fertility of spermatozoa from males exposed to ethylene glycol monomethyl ether. Reprod Toxicol 1990; 4: 21-7.

36 Holloway AJ, Moore HD, Foster PM. The use of in vitro fertilization to detect reductions in the fertility of male rats exposed to 1,3-dinitrobenzene. Fundam App/ Toxicol 1990; 14: 113-22.

37 Fraser LR. In vitro capacitation and fertilization. Methods Enzymol 1993; 225: 23953

38 Moore HD. In vitro manipulation of gametes and embryos for evaluating the effect of chemicals on germ cell function and developmental potential. Food Chem Toxicol 1986; 24: 607-13.

39 Byrd W, Tsu J, Wolf DP. Kinetics of spontaneous and induced acrosomal loss in human sperm incubated under capacitating and noncapacitating conditions. Gamete Res 1989; 22: 109-22.

40 Marquez B, Suarez SS. Different signaling pathways in bovine sperm regulate capacitationand hyperactivation. Biol Reprod 2004; 70: 1626-33.

41 Umemoto Y, Sasaki S, Kojima Y, Kubota H, Kaneko T et al. Gene transfer to mouse testes by electroporation and its influence on spermatogenesis. J Androl 2005; 26 . 264-71.

42 Dhup S, Majumdar SS. Transgenesis via permanent integration of genes in repopulating spermatogonial cells in vivo. Nat Methods 2008; 5: 601-3.

43 Niu Y, Liang S. Progress in gene transfer by germ cells in mammals. J Genet Genom 2008; 35: 701-14.

44 Lishko PV, Botchkina IL, Kirichok Y. Progesterone activates the principal $\mathrm{Ca}^{2+}$ channel of human sperm. Nature 2011; 471: 387-91.

45 Strunker T, Goodwin N, Brenker C, Kashikar ND, Weyand I et al. The CatSper channe mediates progesterone-induced $\mathrm{Ca}^{2+}$ influx in human sperm. Nature 2011; 471 382-6.

46 Barratt CL. The mystery is solved-CatSper is the principal calcium channel activated by progesterone in human spermatozoa. Asian J Androl 2011; 13: 351-2.

47 Benoff S, Cooper GW, Hurley I, Mandel FS, Rosenfeld DL et al. The effect of calcium ion channel blockers on sperm fertilization potential. Fertil Steril 1994; 62: 606-17.

$48 \mathrm{Ho} \mathrm{HC}$, Granish KA, Suarez SS. Hyperactivated motility of bull sperm is triggered at the axoneme by $\mathrm{Ca}^{2+}$ and Not cAMP. Dev Biol 2002; 250: 208-17.

49 Yanagimachi R. Requirement of extracellular calcium ions for various stages of fertilization and fertilization-related phenomena in the hamster. Gamete Res 1982 5: 323-44.

50 Suárez SS, Osman RA. Initiation of hyperactivated flagellar bending in mouse sperm within the female reproductive tract. Biol Reprod 1987; 36: 1191-8.

51 Suarez SS, Dai X. Intracellular calcium reaches different levels of elevation in hyperactivated and acrosome-reacted hamster sperm. Mol Reprod Dev 1995; 42 325-33.

$52 \mathrm{Ho} \mathrm{HC}$, Suarez SS. Characterization of the intracellular calcium store at the base of the sperm flagellum that regulates hyperactivated motility. Biol Reprod 2003; 68: 15906 .

53 Suarez SS, Dai X. Hyperactivation enhances mouse sperm capacity for penetrating viscoelastic media. Biol Reprod 1992; 46: 686-91. 